\title{
Polarization Properties of Four-Wave Mixing in Strained Semiconductor Optical Amplifiers
}

\author{
Roberto Paiella, Guido Hunziker, Jianhui Zhou, Kerry J. Vahala, Member, \\ IEEE, Uzi Koren, Member, IEEE, and Barry I. Miller, Member, IEEE
}

\begin{abstract}
We present a theoretical and experimental study of the polarization properties of the four-wave mixing susceptibility of highly-strained multiquantum-well optical amplifiers and show how the intensity and polarization of the four-wave mixing signal depend on the polarization of the input waves. We demonstrate the validity of our model by generating a wavelength-converted signal having a polarization orthogonal to that of the pump wave at the output of the amplifier. In addition, we discuss the possibility of making the conversion efficiency independent of the input signal polarization by proper selection of the pump polarization.
\end{abstract}

$\mathbf{F}$ OUR-WAVE MIXING (FWM) in semiconductor optical amplifiers (SOA's) [1]-[4] is a promising candidate for the implementation of wavelength conversion [3], [5], [6] in future high-speed multichannel lightwave networks. In a typical FWM wavelength conversion device, beating of the input signal with a strong pump wave produces dynamic gain and index gratings through modulation of carrier density (CDM), and through intraband processes such as carrier heating $(\mathrm{CH})$ and spectral hole burning (SHB). The wavelength converted signal is then generated by scattering of the pump wave from these gratings.

In this Letter, we present a simple model explaining the polarization dependence of the FWM susceptibility in highlystrained multiquantum-well SOA's. An important prediction, which is also verified experimentally, is that the conversion efficiency of such devices remains finite even for orthogonallypolarized pump and input signals. We verify our model by showing how it is possible to control the polarization of the wavelength-converted signal. Finally, we discuss the feasibility of making the FWM conversion efficiency independent of the input signal polarization.

Several density matrix calculations of the FWM susceptibility of SOA's (for the case of copolarized waves) are available in the literature [1], [4]. Here, we allow for arbitrary polarizations (which requires explicit inclusion in the equations of

Manuscript received December 5, 1995; revised February 12, 1996. This work was supported by ARPA Contract DAAL 01-94-K-03430 and the National Science Foundation Grant ECS-9 412862.

R. Paiella, G. Hunziker, and K. J. Vahala are with the Department of Applied Physics, Mail Stop 128-95, California Institute of Technology, Pasadena, California 91125 USA.

J. Zhou was with the Department of Applied Physics, Mail Stop 128-95, California Institute of Technology, Pasadena, CA 91125 USA. He is now with AT\&T Bell Laboratories, Holmdel, NJ 07733 USA

U. Koren and B. I. Miller are with the AT\&T Bell Laboratories, Holmdel, NJ 07733 USA.

Publisher Item Identifier S 1041-1135(96)04309-1. motion of the two spin-degenerate conduction- and valenceband states at each $\vec{k}$ ), and otherwise follow the treatment of [4]. The FWM susceptibility tensor, defined so that the polarization density at the converted signal frequency is $P_{i}^{s}=$ $\chi_{i j k l} E_{j}^{p} E_{k}^{p}\left(E_{l}^{q}\right)^{*}$, is then found, assuming parabolic in-plane dispersion relations (i.e., neglecting valence-band mixing) to have the general form,

$$
\begin{aligned}
\chi_{i j k l}= & \left(\sum_{c, v} \mu_{i}^{v c} \mu_{j}^{c v}\right)\left(\sum_{c, v} \mu_{k}^{c v} \mu_{l}^{v c}\right)\left(\chi_{\mathrm{CDM}}+\chi_{\mathrm{CH}}\right) \\
& +\left(\sum_{c, v, c^{\prime}, v^{\prime}} \mu_{i}^{v c}\left(\mu_{j}^{c j^{\prime}} \mu_{k}^{c^{\prime} v}+\mu_{j}^{c^{\prime} v} \mu_{k}^{c v^{\prime}}\right) \mu_{l}^{v^{\prime} c^{\prime}}\right) \frac{\chi_{\mathrm{SHB}}}{2}
\end{aligned}
$$

where $c, c^{\prime}\left(v, v^{\prime}\right)$ run over the two conduction-band (valenceband) states at any given $\vec{k}, \mu_{i}^{c v}$ is the $i$ th component of the (dimensionless) dipole moment matrix element between states $c$ and $v$ (in multiples of the envelope functions overlap integral times the Kane matrix element), and $i=1$ and 2 denote the TE and TM directions, respectively. Finally, $\chi_{\mathrm{CDM}}, \chi_{\mathrm{CH}}$, and $\chi_{\mathrm{SHB}}$ are identical to the corresponding contributions to the (scalar) FWM susceptibility of a perfectly isotropic medium and are given in [4] as functions of the detuning frequency and of all relevant material parameters. An important point to make is that (1) assumes that all the processes leading to FWM via SHB have the same dephasing rates. ${ }^{1}$

Here, we restrict our discussion to the case of an alternatingstrain multiquantum-well SOA, such as the device used in our experiment, consisting of three pairs of $1 \%$ tensile and compressive InGaAs wells and designed to have polarization independent gain. For such large amounts of strain, and for operation near the band edges, neglect of valence-band mixing is justified. One can then take the total angular momentum eigenstates $|3 / 2, \pm 3 / 2\rangle$ and $|3 / 2, \pm 1 / 2\rangle$ as the Bloch-periodic parts of the valence-band states in the compressive and tensile wells, respectively. Using the well-known selection rules for dipole transitions, one easily finds that the FWM susceptibilities of tensile and compressive wells are subject to the selection rules

$$
\chi_{i j k l}^{T}=\delta_{i j} \delta_{k l} \chi_{i i k k}^{T}+\delta_{i k} \delta_{j l} \chi_{i j i j}^{T}+\delta_{i l} \delta_{j k} \chi_{i k k i}^{T},
$$

${ }^{1}$ In fact, the SHB processes leading to the off-diagonal components $\chi_{i k i k}$ and $\chi_{i k k i}(i \neq k)$ have faster dephasing rates, so that these contributions are somewhat overestimated in (1) and (2). Since these corrections are not essential to the bulk of this letter, they will be presented elsewhere. Furthermore, in highly birefringment SOA's, phase mismatch may be a significant problem for the processes contributing to $\chi_{i k k i}(i \neq k)$. 
and

$$
\chi_{i j k l}^{C}=\delta_{i 1} \delta_{j 1} \delta_{k 1} \delta_{l 1} \chi_{1111}^{C}
$$

respectively, where

$$
\begin{aligned}
\chi_{i i k k}^{T} & =\frac{1}{9}\left[\begin{array}{cc}
1 & 4 \\
4 & 16
\end{array}\right]\left(\chi_{\mathrm{CDM}}^{T}+\chi_{\mathrm{CH}}^{T}+\frac{1}{2} \chi_{\mathrm{SHB}}^{T}\right), \\
\chi_{i k i k}^{T} & =-\chi_{i k k i}^{T}=\frac{2}{9} \chi_{\mathrm{SHB}}^{T} \quad \forall i \neq k, \\
\chi_{1111}^{C} & =\chi_{\mathrm{CDM}}^{C}+\chi_{\mathrm{CH}}^{C}+\frac{1}{2} \chi_{\mathrm{SHB}}^{C}
\end{aligned}
$$

where, in the first line, the matrix rows are labeled by the index $i$, its columns by the index $k$. The same selection rules hold in the presence of nonnegligible valence-band mixing (but with different relative values of the nonzero components of $\chi_{i j k l}$ ).

Finally, the converted signal field at the SOA output $\vec{E}^{s}(L)$ can be determined using expression (2) for the nonlinear susceptibility in the standard coupled mode equations of FWM [1]. The general solution has the form,

$$
\begin{aligned}
E_{i}^{s}(L)=E^{p 2} E^{q *} & {\left[\sum_{k=1}^{2}\left(p_{i} M_{i i k k} p_{k} q_{k}^{*}\right)+\left.M_{i l i l}\right|_{l \neq i} p_{l} p_{i} q_{l}^{*}\right.} \\
& \left.+\left.M_{i l i i}\right|_{l \neq i} p_{l}^{2} q_{i}^{*}\right]
\end{aligned}
$$

where $p_{i}$ and $E^{p}\left(q_{i}\right.$ and $\left.E^{q}\right)$ are the $i$ th component of the polarization unit vector of the pump (signal) and its field amplitude, at the SOA input. In addition, $M_{i j k l}=\left(\chi_{i j k l}^{T}+\right.$ $\left.\chi_{i j k l}^{C}\right) R_{i j k l}$ where $R_{i j k l}$ is a propagation factor whose explicit form is not important here.

The above selection rules for $\chi_{i j k l}$ lend themselves to the following interpretation. The CDM and $\mathrm{CH}$ gratings can only be induced through beating of the same components of the pump and input signal ( $k$ and $l$ either both TE or both TM). The TE (TM) component of the pump can then be scattered by these gratings into a converted signal only along the TE (TM) direction. This is not true in the case of SHB, where both grating formation and pump scattering can also involve orthogonal field components.

These results suggest a simple physical explanation of the polarization dependence of the FWM conversion efficiency. Consider first the sub-TeraHertz detuning range, where the SHB contribution is small, and the last two terms in (3) (proportional to $\chi_{\mathrm{SHB}}^{T}$ ) can be neglected. Each component of the converted signal is then generated by scattering of the same pump component from two "types" of gratings, those formed by the TE components of the input waves ("TEinduced gratings"), and those formed by the TM components ("TM-induced gratings"). The relative phase between the two types of gratings, and hence the degree to which the resulting contributions to $E_{i}^{s}$ add up, depends on the input polarizations. In particular, as illustrated in the insets of Fig. 1, if the pump and input signal have parallel polarizations, the TE- and TMinduced gratings are in phase with each other, so that their contributions to the converted signal field add constructively and the conversion efficiency is maximum. On the other hand, if the input polarizations are orthogonal, the two types of gratings are out of phase and tend to cancel each other. However, since the amplitudes of the two contributions are unequal $\left(M_{i i 11} \neq M_{i i 22}\right)$, this cancellation is incomplete.

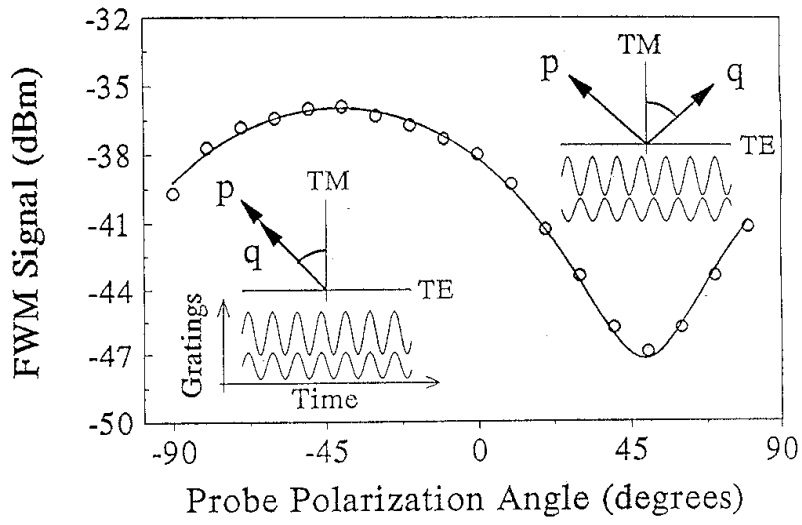

Fig. 1. Converted signal power as a function of the linear polarization angle of the input signal relative to the TM direction, with linearly polarized pump at $-45^{\circ}$ and $1.5-\mathrm{nm}$ detuning. The continuous line is a theoretical fit to (3), with fitting parameters $M_{i i k k}$. The insets show the formation of the dynamic gratings through beating of TE and TM components of pump and input signal. The TE-induced gratings and the TM-induced gratings are in phase for parallel input polarizations and out of phase for orthogonal input polarizations.

As a result, unlike the case of a perfectly isotropic medium, in general the converted signal power is finite even for orthogonally polarized pump and input signal.

This prediction is confirmed by the data shown in Fig. 1, where we plot converted signal power as a function of the angle of the input signal linear polarization with a linearly polarized pump at $-45^{\circ}$ (all angles are relative to the growth axis). A tunable fiber ring laser and a DFB laser, followed by an erbium-doped fiber preamplifier, were used for the pump and input signal, and the SOA output waves were measured using an HP 70950A optical spectrum analyzer. The inputsignal/pump detuning was chosen to be small enough $(1.5 \mathrm{~nm})$ so that the difference in the birefringence experienced by the two input waves is small and thus the angle of polarization between them remains essentially constant throughout the SOA. This ensures that the relatively weak but well defined signal observed for orthogonal input polarizations results from the asymmetry in the susceptibilities embodied in (2).

The reduction in signal power from the optimum case of parallel input polarizations is approximately $11 \mathrm{~dB}$, which is larger than predicted by (3) ( $6 \mathrm{~dB}$ assuming all $R_{i j k l}$ 's equal). We attribute this discrepancy to the strong coupling between neighboring, oppositely strained wells (recently observed in a similar SOA [7]), mediated by several mechanisms such as carrier diffusion, drift and phonon assisted tunneling. Inclusion of interwell transport in the above analysis leads to additional terms to $M_{i i k k}$ of the form $\left(\chi_{i i k k}^{T \leftarrow C}+\chi_{i i k k}^{C \leftarrow T}\right) R_{i i k k}$, where for instance $\chi_{i i k k}^{T \leftarrow C}$ describes a FWM process in which the gratings are formed in a compressive well and transferred to a tensile well where the pump is then scattered. The net result of such terms is to make all the $M_{i i k k}$ 's comparable in magnitude and hence reduce the conversion efficiency for orthogonal input polarizations. Physically, this reduction can be described by noting that the gratings are mainly TM-induced in tensile wells and TE-induced in compressive wells (due to the predominantly TM gain of tensile wells and exclusively TE gain of compressive wells). As a result, when the input 


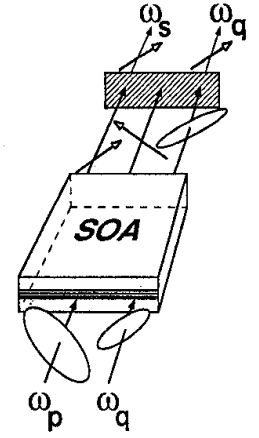

(a)

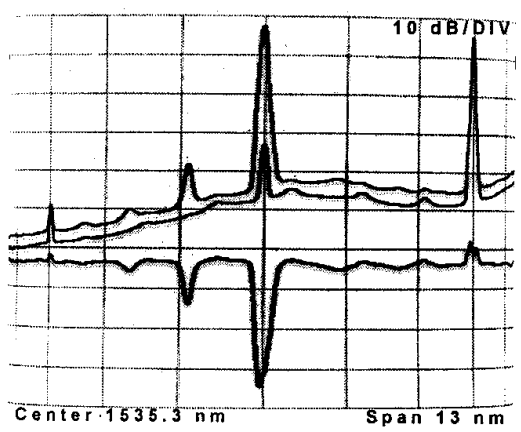

(b)
Fig. 2. (a) Illustration of the pump suppression experiment: the input signal and pump have polarization unit vectors $0.78 \exp i 66^{\circ} \vec{\varepsilon}_{1}+0.63 \vec{\varepsilon}_{2}$, and $0.71\left(\vec{\varepsilon}_{1}+\exp i 98^{\circ} \vec{\varepsilon}_{2}\right)$; correspondingly, at the SOA output, the converted signal and pump are linearly polarized at $+45^{\circ}$ and $-45^{\circ}$, respectively. (b) SOA output spectrum with the above input polarizations and with 4-nm detuning, before (upper trace) and after (middle trace) a linear polarizer at $+45^{\circ}$. The pump (center peak) is suppressed by more than $30 \mathrm{~dB}$ by the polarizer, whereas the converted signal (left peak) is essentially unattenuated. The lower trace is the ratio of the upper two.

waves are orthogonally polarized, the gratings in neighboring wells are out of phase, and coupling of such gratings from one well to another causes an overall cancellation, and hence smaller conversion efficiency.

Finally, in the TeraHertz detuning range SHB becomes the dominant FWM mechanism, and the last two terms in (3) are no longer negligible. These terms introduce a contribution from gratings induced by the TE component of the pump and the TM component of the input signal (or viceversa). Notice that the phase relation between this contribution and those from TE- and TM-induced gratings depends not only on the polarization angle between pump and input signal, but also on their absolute polarization.

The model presented here also provides knowledge on how the polarization of the converted signal depends on that of the input waves. To illustrate, we selected the input polarizations such that the pump and converted signal exit the SOA output linearly polarized at $-45^{\circ}$ and $+45^{\circ}$, respectively. The nonzero components of $M_{i j k l}$ were determined by fitting sets of data such as that in Fig. 1 to (3), for different pump polarizations. The result of our measurement is shown in Fig. 2(b), where the peaks shown are, from right to left, input signal, pump, (pump DFB sidemode), and converted signal (the detuning is $4 \mathrm{~nm}$ ). The upper scan was taken directly at the SOA output, whereas for the middle scan a linear polarizer set at $+45^{\circ}$ was placed between the SOA and the detection stage (the lower trace is the ratio of the first two). Notice that the pump is suppressed by more than $30 \mathrm{~dB}$, while the FWM signal is essentially unattenuated by the polarizer. This idea may be considered as a scheme for pump suppression in wavelength conversion applications, although it has the disadvantage of requiring control of the input signal polarization. Furthermore, the ability to control the polarization of the FWM signal, illustrated with this example, is of importance in optical switching applications [8].

Finally, we consider the possibility of making the FWM conversion efficiency independent of the polarization of the input signal [6], [9] (which is typically random due to the nonpolarization-maintaining character of standard fiber), by proper selection of the pump polarization. From (3), the converted signal power has the form $\left|A q_{1}\right|^{2}+\left|B q_{2}\right|^{2}+$ Re $\left[C q_{1}^{*} q_{2}\right]$, where the coefficients $A, B, C$ depend on the input pump polarization and on the tensor components $M_{i j k l}$. Given the normalization condition $\left|q_{1}\right|^{2}+\left|q_{2}\right|^{2}=1$, this is independent of $q_{i}$ for pump polarization such that $|A|=|B|$ and $\operatorname{Re}[C]=\operatorname{Im}[C]=0$. However, these three equations admit a solution for $p_{1}, p_{2}$ (two independent unknowns) only for a restricted set of values of the $M_{i j k l}$ 's. A simple case is that of all off-diagonal components equal to zero, so that $C=0$ regardless of the input pump polarization which can then be set to make $|A|=|B|$. Physically, this corresponds to each component of the converted signal being generated by scattering from only one type of grating, so that the converted signal power no longer depends on the relative phase between different gratings. The practical implementation of this condition is now under investigation. Alternatively, one may consider use of two nondegenerate pumps at frequencies $\omega_{p}^{(1)}$ and $\omega_{p}^{(2)}$ (this approach has been used in fibers and bulk SOA's in [9]). Then, the conditions $|A|=|B|$ and $\operatorname{Re}[C]=$ $\operatorname{Im}[C]=0$ for polarization independence of the FWM signal at frequency $\omega_{p}^{(1)}+\omega_{p}^{(2)}-\omega_{q}$ depend on the polarization unit vectors of both pumps (four independent unknowns) and are then expected to have solutions for arbitrary sets of the material parameters.

\section{ACKNOWLEDGMENT}

The authors would like to acknowledge R. M. Jopson for coating the device used in these experiments, and D. Geraghty and R. B. Lee for stimulating discussions.

\section{REFERENCES}

[1] G. P. Agrawal, "Population pulsations and nondegenerate four-wave mixing in semiconductor lasers and amplifiers," J. Opt. Soc. Amer. B, vol. 5, pp. 147-158, 1988.

[2] J. Zhou, N. Park, J. W. Dawson, K. J. Vahala, M. A. Newkirk, and B. I. Miller, "Terahertz four-wave mixing spectroscopy for study of ultrafast dynamics in a semiconductor optical amplifier," Appl. Phys. Lett., vol. 63, pp. $1179-1181,1993$

[3] version using semiconductor traveling-wave amplifiers," IEEE Photon. Technol. Lett., vol. 6, pp. 50-52, 1994.

[4] A. Uskov, J. Mørk, and J. Mark, "Wave mixing in semiconductor laser amplifiers due to carrier heating and spectral hole burning," IEEE $J$. Quantum Electron., vol. 30, pp. 1769-1781, 1994.

[5] M. C. Tatham, G. Sherlock, and L. D. Westbrook, " $20 \mathrm{~nm}$ wavelength conversion using nondegenerate four-wave mixing," IEEE Photon. Technol. Lett., vol. 5, pp. 1303-1306, 1993.

[6] R. Schnabel, U. Hilbk, Th. Hermes, P. Meisner, Cv.Helmolt, K. Magalit, F. Raub, W. Pieper, F. J. Westphal, R. Ludwig, L. Kuller, and H. G. Weber, "Polarization insensitive frequency conversion of a $10-$ channel OFDM signal using four-wave-mixing in a semiconductor laser amplifier," IEEE Photon. Technol. Lett., vol. 6, pp. 56-58, 1994.

[7] J. Zhou, N. Park, K. J. Vahala, M. A. Newkirk, and B. I. Miller, "Study of inter-well carrier transport by terahertz four-wave mixing in an optical amplifier with tensile and compressively strained quantum wells," Appl. Phys. Lett., vol. 65, pp. 1897-1899, 1994.

[8] D. Nesset, M. C. Tatham, L. D. Westbrook, and D. Cotter, "Degenerate wavelength operation of an ultrafast all-optical AND gate using four wave mixing in a semiconductor laser amplifiers," Electron. Lett., vol. 30, pp. 1938-1940, 1994.

[9] R. M. Jopson, and R. E. Tench, "Polarization-independent phase conjugation of lightwave signals," Electron. Lett., vol. 29, pp. 2216-2217, 1993. 